\title{
Evolution of helimagnetic correlations when approaching the quantum critical point of $\mathrm{Mn}_{1-x} \mathrm{Fe}_{x} \mathrm{Si}$
}

\author{
C. Pappas $\odot,{ }^{1, *}$ A. O. Leonov, ${ }^{2}$ L. J. Bannenberg $\odot,{ }^{1}$ P. Fouquet $\odot,{ }^{3}$ T. Wolf, ${ }^{4}$ and F. Weber $\odot^{4,5}$ \\ ${ }^{1}$ Faculty of Applied Sciences, Delft University of Technology, Mekelweg 15, $2629 \mathrm{JB}$ Delft, The Netherlands \\ ${ }^{2}$ Department of Chemistry, Faculty of Science, Hiroshima University Kagamiyama, Higashi Hiroshima, Hiroshima 739-8526, Japan \\ ${ }^{3}$ Institut Laue Langevin, 71 Avenue des Martyrs, CS 20156, Grenoble, France \\ ${ }^{4}$ Institute for Solid State Physics, Karlsruhe Institute of Technology, 76131 Karlsruhe, Germany \\ ${ }^{5}$ Institute for Quantum Materials and Technologies, Karlsruhe Institute of Technology, 76131 Karlsruhe, Germany
}

(Received 7 May 2020; revised 3 November 2020; accepted 11 December 2020; published 8 January 2021)

\begin{abstract}
We present a comprehensive investigation of the evolution of helimagnetic correlations in $\mathrm{Mn}_{1-x} \mathrm{Fe}_{x} \mathrm{Si}$ with increasing doping. By combining polarized neutron scattering and high resolution neutron spin echo spectroscopy we investigate three samples with $x=0.09,0.11$, and 0.14 , i.e., with compositions on both sides of the concentration $x^{*} \sim 0.11$ where the helimagnetic Bragg peaks disappear and between $x^{*}$ and the quantum critical concentration $x_{C} \sim 0.17$, where $T_{C}$ vanishes. We find that the abrupt disappearance of the long range helical periodicity at $x^{*}$ does not affect the precursor fluctuating correlations. These build up with decreasing temperature in a similar way as for the parent compound $\mathrm{MnSi}$. Also the dynamics bears strong similarities to $\mathrm{MnSi}$. The analysis of our results indicates that frustration, possibly due to achiral Ruderman-Kittel-Kasuya-Yosida (RKKY) interactions, increases with increasing Fe doping. We argue that this effect explains both the expansion of the precursor phase with increasing $x$ and the abrupt disappearance of long range helimagnetic periodicity
\end{abstract} at $x^{*}$.

DOI: 10.1103/PhysRevResearch.3.013019

\section{INTRODUCTION}

The physics of the chiral magnet MnSi touches several fundamental questions in condensed-matter physics, from the stabilization of exotic states like chiral skyrmions [1] to the interplay between localized and itinerant magnetism [2-8] as well as to non-Fermi-liquid behavior [9-14] and quantum fluctuations [15-23]. Under pressure a non-Fermi-liquid behavior sets in without quantum criticality [11,15,24,25]. Furthermore, the first-order transition temperature $T_{C}$ is driven to $0 \mathrm{~K}$ at $p_{C} \sim 1.4 \mathrm{GPa}$, although the magnetic moment does not vanish. In the region of the temperature-pressure phase diagram where $p \gtrsim p_{C}$ and $T_{C}=0 \mathrm{~K}$, long range range spiral and skyrmion correlations are restored under magnetic fields [7], a result that has been attributed to a softening of the magnetic moment. Pressure would therefore enhance the itinerant electron character of magnetism, triggering the suppression of $T_{C}$. On the other hand, in the absence of quantum critical point $(\mathrm{QCP})$ at $p_{C}$ [19], the origin and nature of the highly debated non-Fermi-liquid phase [16-18,20,26,27] remains an open question. It was suggested that this phase, out of which magnetic fields induce long range spiral correlations, is fluctuating and possibly of quantum nature [19]. However,

\footnotetext{
*c.pappas@tudelft.nl

Published by the American Physical Society under the terms of the Creative Commons Attribution 4.0 International license. Further distribution of this work must maintain attribution to the author(s) and the published article's title, journal citation, and DOI.
}

our high resolution neutron spin echo (NSE) spectroscopy measurements did not reveal the existence of such fluctuations, possibly due to limitations (background contribution of the pressure cell) inherent to measurements under high pressures [7].

These limitations are overcome by chemical pressure, in the form of $\mathrm{Fe}$ doping in $\mathrm{Mn}_{1-x} \mathrm{Fe}_{x} \mathrm{Si}$. The behavior of this system resembles that of MnSi under pressure [28-37], and our magnetization, susceptibility, and SANS investigations $[32,33]$ led to the phase diagram shown in Fig. 1. With increasing doping, the transition temperature decreases continuously and vanishes at $x_{C} \sim 0.17$. On the other hand, the helimagnetic Bragg peaks, a signature of long range helimagnetic periodicity, disappear abruptly at a much lower concentration of $x^{*} \sim 0.11$. For $x \gtrsim x^{*}$ magnetic susceptibility and electric transport phenomena reveal a non-Fermi-liquid behavior, which, as in $\mathrm{MnSi}$ under pressure, has been attributed to a chiral spin liquid state governed by quantum fluctuations [35-37]. Here we investigate the evolution of helimagnetic correlations and their dynamics as a function of chemical substitution in $\mathrm{Mn}_{1-x} \mathrm{Fe}_{x} \mathrm{Si}$ using polarized neutron scattering and neutron spin echo (NSE) spectroscopy. We investigated three samples with $x=0.09,0.11$ and 0.14 , i.e., with compositions on both sides of $x^{*}$ and between $x^{*}$ and $x_{C}$. Our results reveal that the abrupt disappearance of long range helimagnetic periodicity at $x^{*}$ does not affect the precursor fluctuating correlations. These build up with decreasing temperature in a way that is very similar to that of the parent compound $\mathrm{MnSi}$. Also the dynamics, i.e., the characteristic relaxation times and their temperature dependence, resembles the behavior of $\mathrm{MnSi}$. 


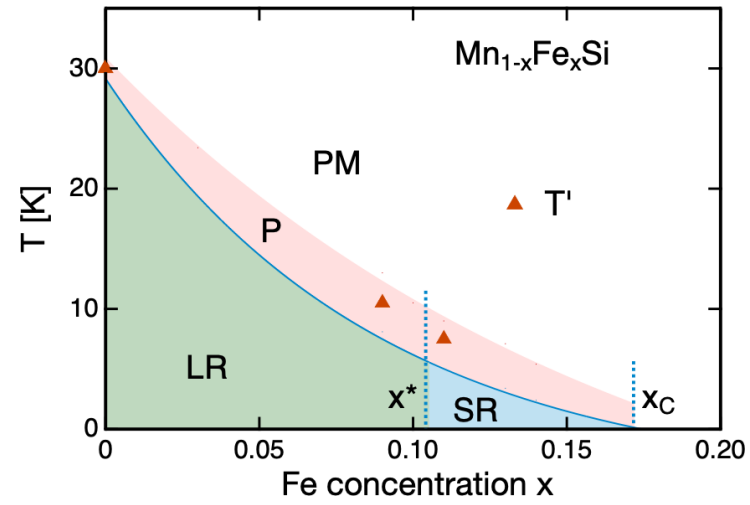

FIG. 1. Phase diagram of $\mathrm{Mn}_{1-x} \mathrm{Fe}_{x} \mathrm{Si}$ deduced from our previous SANS and susceptibility measurements [32,33]. The pink shaded area indicates the precursor phase, $\mathrm{P}$, and PM stands for the paramagnetic phase. The blue line indicates the transition to the ordered helimagnetic phase with long (LR) and short (SR) range periodicity respectively. The temperatures $T^{\prime}$ have been determined as shown in Figs. 2(b) and 2(c).

In order to understand these results we discuss the evolution of magnetic interactions with doping and also compare $\mathrm{Mn}_{1-x} \mathrm{Fe}_{x} \mathrm{Si}$ with $\mathrm{MnSi}$ under pressure. Our analysis brings us to the conclusion that in $\mathrm{Mn}_{1-x} \mathrm{Fe}_{x} \mathrm{Si}$ doping introduces frustration. We argue that this effect explains both the destabilization of long range helimagnetic periodicity at $x^{*}$ as well as the robustness of the precursor phase and its expansion with increasing $x$.

\section{EXPERIMENTAL RESULTS}

The samples were grown using the Bridgeman method. For $x=0.09,0.11$ we measured the same single crystals as in our previous SANS study [32]. For $x=0.14$ and in order to compensate for the neutron intensity losses at this high Fe doping we chose a large polycrystalline and racemate polycrystalline sample, from the same batch as the samples of our previous studies [32,33]. On one hand, this choice is not problematic, since at this composition the magnetic Bragg peaks have disappeared and the magnetic correlations are no longer pinned to the lattice. On the other hand, as it will be explained below, the polarized neutron scattering from this sample cannot be used to determine the degree of chirality of the magnetic correlations.

The measurements were performed at the IN11 spectrometer, of the Institut Laue Langevin, using the paramagnetic NSE configuration and an incoming wavelength of $0.55 \mathrm{~nm}$. For each sample we determined the polarized neutron scattering and the NSE spectra at the respective maxima of the magnetic scattering intensity. These occur at the scattering vector values $Q=\tau=0.58,0.68$ and $0.88 \mathrm{~nm}^{-1}$ leading to helical pitches $\ell=10.8,9.2$, and $7.1 \mathrm{~nm}$, for $x=0.09,0.11$, and 0.14 respectively, in good agreement with our previous SANS results [32].

By exploiting the polarization analysis capabilities of the experimental setup we obtained an accurate determination of the magnetic scattering and of the chiral fraction of the magnetic correlations [38-43]. The results, shown in

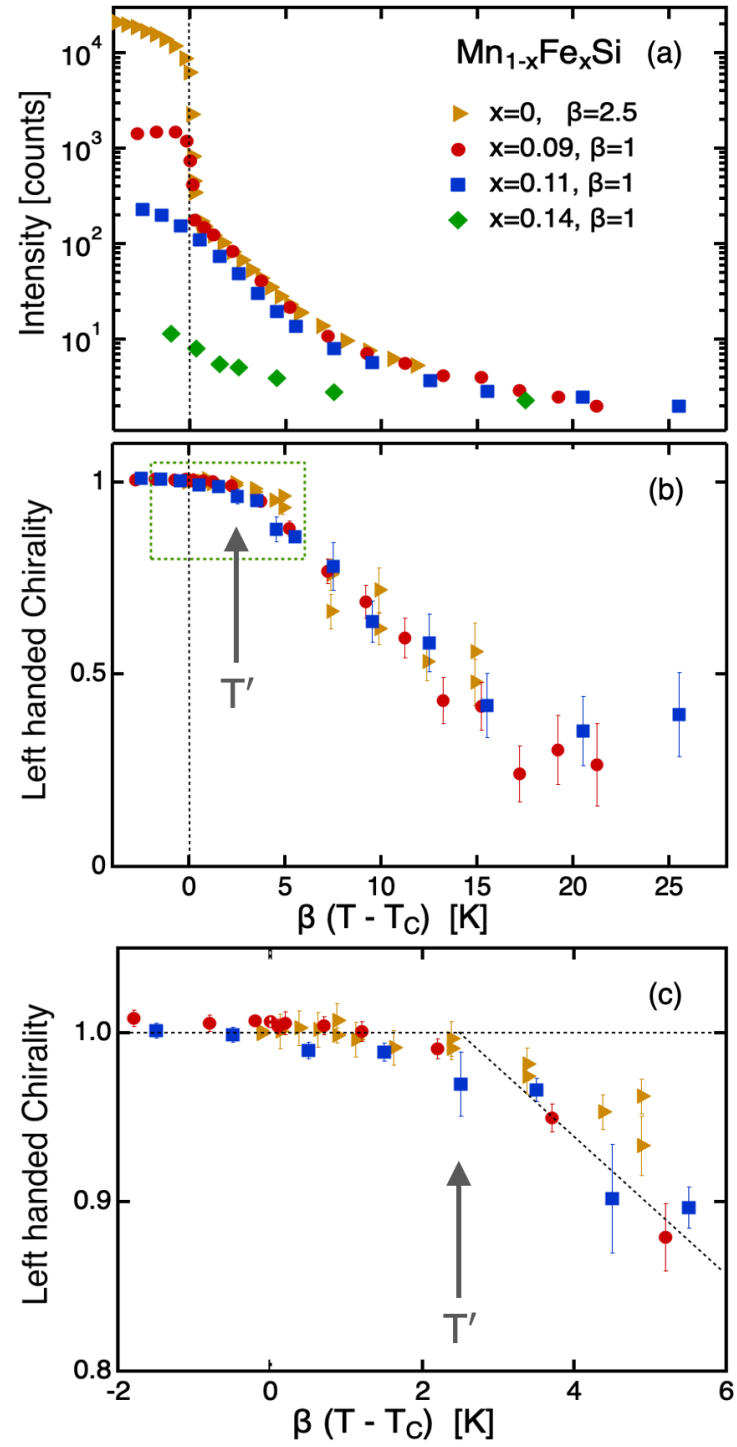

FIG. 2. Temperature dependence of the helimagnetic SANS intensity (a) and the left-handed chiral fraction of the scattering [(b) and (c)]. For the sake of comparison between the results of the three compositions investigated here and the parent compound $\mathrm{MnSi}$ (data from $[38,39]$ ), the abscissa is the scaled temperature difference $\beta\left(T-T_{C}\right)$, with $\beta=2.5$ for $\mathrm{MnSi}$ and $\beta=1$ otherwise. In this way is is possible to account for the broadening of the precursor phase found for the doped samples. $T_{C}=7.8,5$, and $2.5 \mathrm{~K}$ for $x=0.09,0.11$, and 0.14 respectively. Panel (c) shows a close-up view of the green rectangle area shown in (b). The dotted lines in (c) illustrate the determination of $T^{\prime}$. For $T<T^{\prime}$ the scattering of $\mathrm{MnSi}, \mathrm{Mn}_{0.91} \mathrm{Fe}_{0.09} \mathrm{Si}$, and $\mathrm{Mn}_{0.89} \mathrm{Fe}_{0.11} \mathrm{Si}$ reaches full left-handed chirality within at least two times the error bars.

Figs. 2(b) and 2(c), highlight the abrupt change of behavior at $x^{*}$.

For $x=0.09$, i.e., for $x<x^{*}$, a jump of intensity marks the onset of the helimagnetic Bragg peaks and the first-order phase transition at $T_{C}=7.8 \mathrm{~K}$. This jump disappears for $x>$ $x^{*}$. However, for $x=0.11$ the precursor correlations build up with decreasing temperature in the same way as for $x=0.09$. Consequently, the precursor phase builds up in a similar way on both sides of $x^{*}$, a result that is hard to reconcile with the 
Brazovskii scenario proposed to explain the first-order phase transition in $\mathrm{MnSi}$ and other chiral magnets [44].

For $x=0.14$, the evolution of intensity with temperature is much slower and can only be superimposed with data from lower dopings assuming a negative $T_{C}$.

Figures 2(b) and 2(c) show that the scattering from both single crystalline samples, with $x=0.09$ and 0.11 , is fully chiral at low temperatures. Unpublished results on single crystals indicate that the correlations remain chiral for dopings even higher than $x_{C}$ [45]. However, the scattering from our $14 \%$ polycrystalline sample was achiral. This brought us to the conclusion that it is a racemate, combining grains with different structural chiralities, because in this system structural and magnetic chiralities are coupled [46]. Thus, the polarized neutron scattering from this sample does not reflect the chirality of magnetic correlations, and for this reason this concentration is not included in Figs. 2(b) and 2(c).

For $x=0.09$ and 0.11 , full chirality extends up to $T^{\prime} \sim$ $T_{C}+2.5 \mathrm{~K}$, thus well above $T_{C}$. The temperature interval $T^{\prime}-T_{C}$ is $\sim 2.5$ times broader than in $\mathrm{MnSi}$, where full chirality is found up to $T^{\prime} \sim T_{C}+1 \mathrm{~K}[38,39]$. In order to account for this difference when comparing the behavior of the pristine and doped samples, the abscissas in Fig. 2 are the scaled temperature differences $\beta\left(T-T_{C}\right)$, with $\beta=2.5$ for $\mathrm{MnSi}$ and $\beta=1$ otherwise. In these plots $\mathrm{Mn}_{0.91} \mathrm{Fe}_{0.09} \mathrm{Si}$ reproduces the behavior of $\mathrm{MnSi}$, for both the intensity and the chiral fraction.

Further insight in the effect of chemical doping on the magnetic behavior is provided by the intermediate scattering functions $I(Q=\tau, t)$, determined by NSE spectroscopy and shown in Fig. 3. The spectra follow an exponential decay that can be fitted by the function $I(Q=\tau, t)=(1-$ $\left.a_{e l}\right) \exp \left(-t / t_{0}\right)+a_{e l}$, where $t_{0}$ is the characteristic relaxation time and $a_{e l}$ the elastic fraction of the scattering. This behavior contrasts with that of other disordered helimagnets, such as $\mathrm{Fe}_{0.7} \mathrm{Co}_{0.3} \mathrm{Si}[47,48]$ or $\mathrm{Zn}$ doped $\mathrm{Cu}_{2} \mathrm{OSeO}_{3}$ [49], where strong deviations from exponentiality have been reported.

The exponential relaxation rules out a spin-glass-like ground state, the footprint of which would have been a stretched exponential decay [50,51]. Furthermore, the NSE spectra become completely elastic at the base temperature, a result which excludes the spin liquid scenario suggested for $x>x^{*}$ [35-37]. The elastic fraction, depicted in Fig. 4(a), reflects the evolution of the scattered intensity shown in Fig. 2(b). The change at $T_{C}$ is almost step-like, characteristic of the first-order phase transition, for $\mathrm{MnSi}$ and $\mathrm{Mn}_{0.91} \mathrm{Fe}_{0.09} \mathrm{Si}$. On the other hand, for $x>x^{*}, a_{e l}$ increases gradually with decreasing temperature, as also found in the disordered helimagnet $\mathrm{Fe}_{0.7} \mathrm{Co}_{0.3} \mathrm{Si}$ [47].

The characterisitic relaxation times, $t_{0}$, depicted in Figs. 4(b) and 4(c), do not change with doping and are comparable to those of $\mathrm{MnSi}[38,39]$. Their values vary between 0.1 and $2 \mathrm{~ns}$, leading to characteristic energies, $\hbar \omega$, between 6.58 and $0.33 \mu \mathrm{eV}$. These values are much lower than those reported by a previous study [30], which, however, suffered from a low $Q$ and energy resolution. The energies found here correspond to temperatures between 80 and $4 \mathrm{mK}$. Consequently, these are classical fluctuations, with $\hbar \omega \ll k T$, not the quantum fluctuations discussed in the literature [35-37]. Thus, classical critical slowing down governs the dynamics

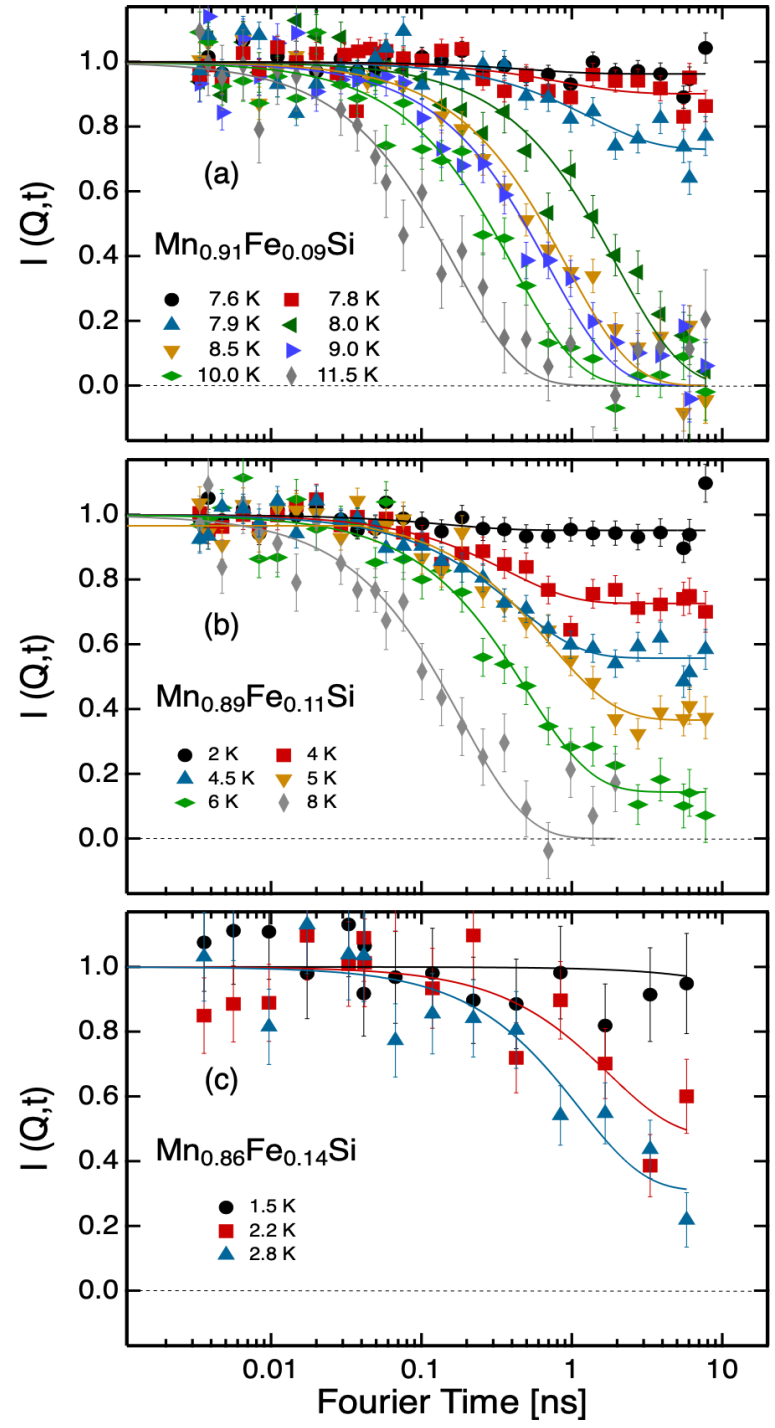

FIG. 3. Intermediate scattering functions, determined by neutron spin echo spectroscopy, of $\mathrm{Mn}_{0.91} \mathrm{Fe}_{0.09} \mathrm{Si}$ (a), $\mathrm{Mn}_{0.89} \mathrm{Fe}_{0.11} \mathrm{Si}$ (b), and $\mathrm{Mn}_{0.86} \mathrm{Fe}_{0.14} \mathrm{Si}$ (c). The lines represent fits to an exponential decay (see text).

of helimagnetic correlations for $x>x^{*}$, masking the quantum criticality associated with the putative $\mathrm{QCP}$ at $x_{C}$.

\section{DISCUSSION}

Our results show that Fe doping affects the first-order transition and the long range helical periodicity in a very different way than the precursor phase. While the former disappear abruptly at $x^{*}$, the latter expands and persists up to higher dopings. Also the characteristic relaxation times of the fluctuations are comparable to those found in $\mathrm{MnSi}$. In order to understand these results and in particular the robustness of the precursor phase, we adopt the Dzyaloshinskii model for cubic noncentrosymmetric ferromagnets [52-54], which leads to the free energy per unit cell [55]

$$
\mathcal{E}=\frac{J a^{2}}{2} \sum_{i=x, y, z} \partial_{i} \boldsymbol{m} \cdot \partial_{i} \boldsymbol{m}+\text { Da } \boldsymbol{m} \cdot \nabla \times \boldsymbol{m}-a^{3} \mu_{0} M \boldsymbol{m} \cdot \boldsymbol{H}+\mathcal{E}_{a},
$$



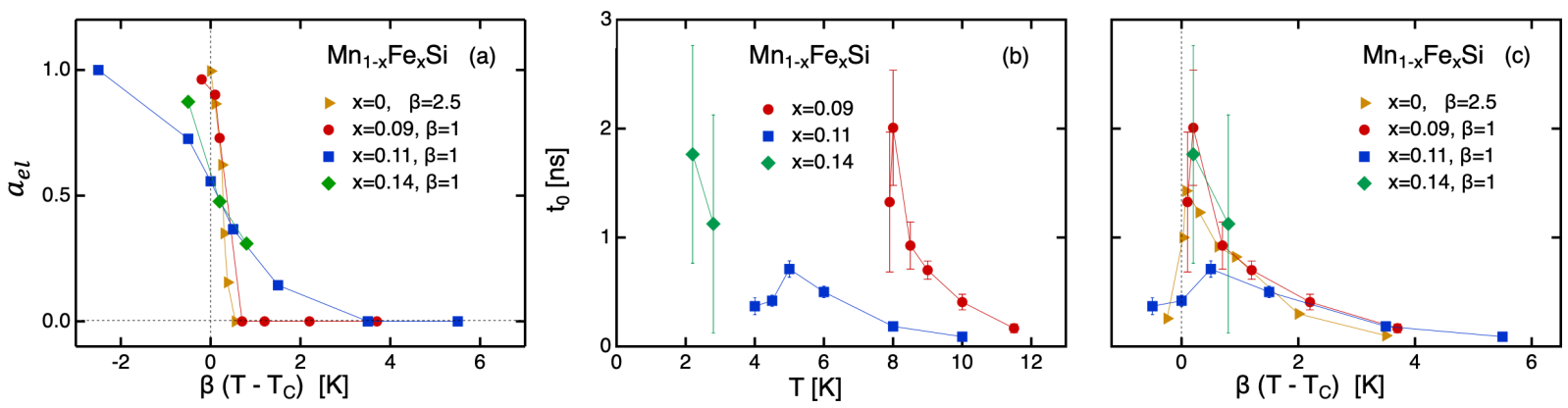

FIG. 4. Evolution with temperature and doping of the elastic fractions $a_{e l}$ and the characteristic times $t_{0}$ deduced from fitting an exponential decay to the NSE spectra of Fig. 3 (see text). In (a) and (c) the evolution of $a_{e l}$ and $t_{0}$ is compared with that of parent compound MnSi (data from [38,39]) and for this reason the abscissa is, as in Fig. 2, the scaled temperature difference $\beta\left(T-T_{C}\right)$, with $\beta=2.5$ for MnSi and $\beta=1$ otherwise.

with $J$ the ferromagnetic Heisenberg exchange, $D$ the Dzyaloshinskii-Moriya (DMI) interaction, $\boldsymbol{m}$ the unit vector in the direction of the magnetization, $M$ the magnetization, $a$ the lattice constant, and $\mathcal{E}_{a}$ the magnetic anisotropy energy. The ground state consists of helical spirals with a periodicity of $\ell=2 \pi a J / D$ propagating along specific crystallographic directions fixed by magnetic anisotropy. A magnetic field strong enough to overcome the anisotropy aligns the spirals towards its direction and tilts the magnetic moments by an angle given by $\cos \theta=H / H_{C 2}$, where $H_{C 2}$ is the critical field above which the homogeneous or field-polarized state sets in. The model of Eq. (1) leads to $\mu_{0} M_{0} H_{C 2}=D^{2} / \mathrm{Ja}^{3}$, with $M_{0}$ the volume magnetization at $H_{C 2}$. Therefore, at low temperatures, the pitch of the helical spirals can be written as $\ell=$ $2 \pi a \sqrt{J /\left(a^{3} \mu_{0} M_{0} H_{C 2}\right)}$ and, by assuming that $J$ is given by the Curie-Weiss temperature, $T_{C W}$ (mean field approximation), it can be derived from quantities that have been determined experimentally (see, e.g., Table 1 of [33]). In particular the lattice constant, $a$, does not change significantly with temperature [11] but varies linearly with $x$, between $0.4565 \mathrm{~nm}$ for $\mathrm{MnSi}$ and $0.449 \mathrm{~nm}$ for FeSi [46].

By combining this experimental input we calculate the values of $\ell$ that are depicted in Fig. 5 and vary very little with doping. For the parent compound $\mathrm{MnSi}$ the relative difference between the experimental and calculated values of $\ell$ does not exceed $6 \%$, which gives confidence to our approach. For the sake of comparison Fig. 5 also shows the pressure dependence of $\ell$ for MnSi (base temperature data from Fig. 2(e) of [7]), which is weak and follows the trend of the calculated values. This contrasts with the behavior of $\mathrm{Mn}_{1-x} \mathrm{Fe}_{x} \mathrm{Si}$, where $\ell$ decreases rapidly with increasing Fe doping. At $x=0.11$, the highest $\mathrm{Fe}$ concentration where it was still possible to determine both $M_{0}$ and $H_{C 2}$, the experimental value of $\ell$ is half the one expected from the model of Eq. (1).

An even more drastic reduction of the helical periodicity was recently found in $\mathrm{MnSi}_{1-x} \mathrm{Ge}_{x}[56,57]$ and has been attributed to frustration [58]. The effect of frustration can be explained by the interplay between the DMI and higher-order derivatives terms in Eq. (1). These were the original skyrmion stabilization mechanism considered by Skyrme [59] and lead to an attracting skyrmion-skyrmion or soliton-soliton interaction [60]. The numerical simulations in [58] reveal a drastic decrease of $\ell$ in the presence of an additional antiferromag- netic exchange $J_{A F}$ term in Eq. (1). This reduction occurs even for weak frustration and, although it was not possible to derive an analytical expression of general validity, the limit $\ell \gg a$, leads to [58]

$$
\ell \approx 2 \pi a \frac{J}{D}\left(1-4 \frac{J_{A F}}{J}\right) .
$$

Following this approximation for $\mathrm{Mn}_{0.89} \mathrm{Fe}_{0.11} \mathrm{Si}$, where $\ell / a \approx 18$, the $50 \%$ pitch reduction leads to $J_{A F} / J \approx 1 / 8$. This relatively low ratio points to weak frustration and is consistent with the weak disorder seen by electron spin resonance [61] and the exponential relaxation of the NSE spectra.

Antiferromagnetic interactions may result from oscillatory Ruderman-Kittel-Kasuya-Yosida (RKKY) exchange between localized magnetic moments, as suggested by the analysis of the ordinary and anomalous Hall effects [62]. These achiral interactions increase with increasing $x$ and this effect would explain the destabilization of the helical periodicity at $x^{*}$. It was suggested that RKKY interactions arise from the modification of the electron and hole concentrations by the substitution of $\mathrm{Mn}$ by $\mathrm{Fe}$ [62]. Thus, similarly to $\mathrm{MnSi}$ under pressure, the modification of the electronic state drives

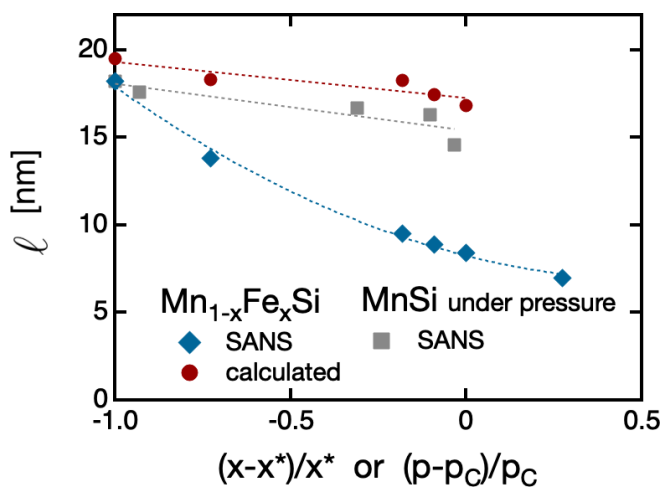

FIG. 5. Evolution of the helical pitch as a function of Fe doping, in the case of $\mathrm{Mn}_{1-x} \mathrm{Fe}_{x} \mathrm{Si}$, or as a function of pressure, in the case of MnSi (base temperature data from [32] and [7] respectively). For the sake of comparison the abscissa is either the relative doping $\left(x-x^{*}\right) / x^{*}$ for $\mathrm{Mn}_{1-x} \mathrm{Fe}_{x} \mathrm{Si}$ or the relative pressure $\left(p-p_{C}\right) / p_{C}$ for $\mathrm{MnSi}$. The red dots have been calculated for $\mathrm{Mn}_{1-x} \mathrm{Fe}_{x} \mathrm{Si}$ using the model of Eq. (1). 
the destabilization of the long range helical order and of the sharp first-order phase transition at $T_{C}$. However, the specific microscopic mechanisms are different for the two systems, as highlighted by the evolution of the helical pitch $\ell$ shown in Fig. 5. Frustration and disorder are important for $\mathrm{Mn}_{1-x} \mathrm{Fe}_{x} \mathrm{Si}$, in contrast to $\mathrm{MnSi}$ under pressure.

Frustration can stabilize spiral and skyrmion periodic states $[60,63]$ but, unlike DMI, it does not impose the chirality, i.e., it does not impose a sense of rotation. This leads to rich phase diagrams, with phases that are impossible in chiral magnets. The competition between frustration and DMI, however, has only been touched upon in the literature $[58,64,65]$ and only in the low-temperature regime with a fixed magnetization modulus. In fact, the influence of frustration on the phase transition and the precursor states in chiral magnets is a most challenging and unresolved problem, which we approach based on general considerations.

Near the ordering temperatures, the functional of Eq. (1) must be supplemented by the Landau expansion in powers of the magnetization [54]:

$$
W_{0}=A\left(T-T_{0}\right) M^{2}+B M^{4},
$$

where $B>0$ and $T_{0}$ is the ferromagnetic ordering temperature, i.e., the Curie temperature in the absence of DMI. Under the influence of temperature and of an applied magnetic field, this term enables variations of the magnetization amplitude, which lead to sizable effects in the vicinity of $T_{C}$, the transition temperature in the presence of DMI. In the precursor region of chiral magnets, the chiral twisting is accompanied by strong longitudinal modulations of the magnetization and its interplay with rotational modes [17,66-68]. Thus, anomalous spin textures can be expected, such as a staggered half-skyrmion lattice [17] - a close analog of the square lattice of merons in frustrated magnets [69]. Even in the absence of frustration these precursor modulated states may have both senses of magnetization rotation. However, the modulus increases or decreases depending on whether the rotation adopts the correct or the wrong rotational sense $[67,70]$ with the correct chirality eventually dominating close to $T_{C}$, as shown in Fig. 2. This mechanism induces fan-like oscillations of isolated skyrmions [67] and solitons [70] and leads to their attracting interaction with potentials containing a plethora of local minima at different mutual distances.

An increasing frustration will amplify these precursor modulations and will thus enhance and expand the precursor region, as is indeed the case for $\mathrm{Mn}_{1-x} \mathrm{Fe}_{x} \mathrm{Si}[28-30,32,33]$.

\section{CONCLUSION}

To conclude, our results shed light on the evolution of helimagnetic correlations and fluctuations in $\mathrm{Mn}_{1-x} \mathrm{Fe}_{x} \mathrm{Si}$ for $x<x_{C}$. On one hand, our observations rule out both the spin liquid and the quantum fluctuations hypothesis for $x>x^{*}$, which cannot be associated with a quantum critical point. On the other hand, our analysis indicates that, with increasing doping, frustration increases, which we attribute to increasing competition between chiral DMI and achiral RKKY interactions. We argue that frustration explains the expansion of the precursor phase for $x<x_{C}$ and the destabilization of the long range heilmagnetic periodicity at $x^{*}$.

More generally, both frustration and DMI can stabilize spiral and skyrmion periodic states, and the competition between the two can lead to rich phase diagrams with new phases and spin configurations, providing a fertile ground for future developments.

Data from this work are available online [71].

\section{ACKNOWLEDGMENTS}

C.P. thanks S. V. Grigoriev for useful discussions and for sharing unpublished experimental results. The experiments were performed at the Institut Laue Langevin, France. The single crystals were aligned at the OrientExpress instrument with the support of Bachir Ouladdiaf. The work of L.J.B. and C.P. has been financially supported by The Netherlands Organization for Scientific Research (NWO) through Project No. 72 1.012.102 (Larmor). C.P. acknowledges travel support from JSPS KAKENHI Grant No. JP15H05882 (J-Physics).
[1] S. Mühlbauer, B. Binz, F. Jonietz, C. Pfleiderer, A. Rosch, A. Neubauer, R. Georgii, and P. Böni, Skyrmion lattice in a chiral magnet, Science 323, 915 (2009).

[2] Y. Ishikawa, G. Shirane, J. A. Tarvin, and M. Kohgi, Magnetic excitations in the weak itinerant ferromagnet MnSi, Phys. Rev. B 16, 4956 (1977).

[3] C. Thessieu, J. Flouquet, G. Lapertot, A. N. Stepanov, and D. Jaccard, Magnetism and spin fluctuations in a weak itinerant ferromagnet: MnSi, Solid State Commun. 95, 707 (1995).

[4] M. Corti, F. Carbone, M. Filibian, Th. Jarlborg, A. A. Nugroho, and P. Carretta, Spin dynamics in a weakly itinerant magnet from ${ }^{29}$ Si NMR in MnSi, Phys. Rev. B 75, 115111 (2007).

[5] S. V. Demishev, A. V. Semeno, A. V. Bogach, V. V. Glushkov, N. E. Sluchanko, N. A. Samarin, and A. L. Chernobrovkin, Is $\mathrm{MnSi}$ an itinerant-electron magnet? Results of ESR experiments, JETP Lett. 93, 213 (2011).
[6] H. Yasuoka, K. Motoya, M. Majumder, S. Witt, C. Krellner, and M. Baenitz, Chiral magnetism in an itinerant helical magnet, MnSi - An extended ${ }^{29}$ Si NMR study, J. Phys. Soc. Jpn. 85, 073701 (2016).

[7] L. J. Bannenberg, R. Sadykov, R. M. Dalgliesh, C. Goodway, D. L. Schlagel, T. A. Lograsso, P. Falus, E. Lelièvre-Berna, A. O. Leonov, and C. Pappas, Skyrmions and spirals in MnSi under hydrostatic pressure, Phys. Rev. B 100, 054447 (2019).

[8] A. Yaouanc, P. Dalmas de Réotier, B. Roessli, A. Maisuradze, A. Amato, D. Andreica, and G. Lapertot, Dual nature of magnetism in MnSi, Phys. Rev. Research 2, 013029 (2020).

[9] C. Pfleiderer, S. R. Julian, and G. G. Lonzarich, NonFermi-liquid nature of the normal state of itinerant-electron ferromagnets, Nature (London) 414, 427 (2001).

[10] N. Doiron-Leyraud, I. R. Walker, L. Taillefer, M. J. Steiner, S. R. Julian, and G. G. Lonzarich, Fermi-liquid breakdown in 
the paramagnetic phase of a pure metal, Nature (London) 425 , 595 (2003).

[11] C. Pfleiderer, P. Böni, T. Keller, U. K. Rößler, and A. Rosch, Non-fermi liquid metal without quantum criticality, Science 316, 1871 (2007).

[12] M. Lee, W. Kang, Y. Onose, Y. Tokura, and N. P. Ong, Unusual Hall Effect Anomaly in MnSi under Pressure, Phys. Rev. Lett. 102, 186601 (2009)

[13] R. Ritz, M. Halder, M. Wagner, C. Franz, A. Bauer, and C. Pfleiderer, Formation of a topological non-Fermi liquid in MnSi, Nature (London) 497, 231 (2013).

[14] T. R. Kirkpatrick and D. Belitz, Generic non-Fermi-liquid behavior of the resistivity in magnets with ferromagnetic, helical, or skyrmionic order, Phys. Rev. B 97, 064411 (2018).

[15] C. Pfleiderer, G. J. McMullan, S. R. Julian, and G. G. Lonzarich, Magnetic quantum phase transition in MnSi under hydrostatic pressure, Phys. Rev. B 55, 8330 (1997).

[16] S. Tewari, D. Belitz, and T. R. Kirkpatrick, Blue Quantum Fog: Chiral Condensation in Quantum Helimagnets, Phys. Rev. Lett. 96, 047207 (2006).

[17] U. K. Rößler, A. N. Bogdanov, and C. Pfleiderer, Spontaneous skyrmion ground states in magnetic metals, Nature (London) 442, 797 (2006).

[18] J. M. Hopkinson and H.-Y. Kee, Origin and consequences of unpinned helical order: Application to $\mathrm{MnSi}$ under pressure, Phys. Rev. B 79, 014421 (2009).

[19] C. Pfleiderer, A. Neubauer, S. Muhlbauer, F. Jonietz, M. Janoschek, S. Legl, R. Ritz, W. Münzer, C. Franz, P. G. Niklowitz, T. Keller, R. Georgii, P. Böni, B. Binz, A. Rosch, U. K. Rößler, and A. N. Bogdanov, Quantum order in the chiral magnet MnSi, J. Phys. Condens. Matter 21, 164215 (2009).

[20] T. R. Kirkpatrick and D. Belitz, Columnar Fluctuations as a Source of Non-Fermi-Liquid Behavior in Weak Metallic Magnets, Phys. Rev. Lett. 104, 256404 (2010).

[21] K.-Y. Ho, T. R. Kirkpatrick, Y. Sang, and D. Belitz, Ordered phases of itinerant Dzyaloshinsky-Moriya magnets and their electronic properties, Phys. Rev. B 82, 134427 (2010).

[22] F. Krüger, U. Karahasanovic, and A. G. Green, Quantum Orderby-Disorder near Criticality and the Secret of Partial Order in MnSi, Phys. Rev. Lett. 108, 067003 (2012).

[23] A. A. Povzner, A. G. Volkov, and T. A. Nogovitsyna, Electronic structure and quantum spin fluctuations at the magnetic phase transition in MnSi, Physica B 536, 408 (2018).

[24] C. Pfleiderer, D. Reznik, L. Pintschovius, H. v Lohneysen, M. Garst, and A. Rosch, Partial order in the non-Fermi-liquid phase of MnSi, Nature (London) 427, 227 (2004).

[25] L. Pintschovius, D. Reznik, C. Pfleiderer, and H. von Löhneysen, Partial magnetic order in the itinerant-electron magnet MnSi, Pramana 63, 117 (2004).

[26] B. Binz, A. Vishwanath, and V. Aji, Theory of the Helical Spin Crystal: A Candidate for the Partially Ordered State of MnSi, Phys. Rev. Lett. 96, 207202 (2006).

[27] I. Fischer, N. Shah, and A. Rosch, Crystalline phases in chiral ferromagnets: Destabilization of helical order, Phys. Rev. B 77, 024415 (2008).

[28] A. Bauer, A. Neubauer, C. Franz, W. Münzer, M. Garst, and C. Pfleiderer, Quantum phase transitions in single-crystal $\mathrm{Mn}_{1-x} \mathrm{Fe}_{x} \mathrm{Si}$ and $\mathrm{Mn}_{1-x} \mathrm{Co}_{x} \mathrm{Si}$ : Crystal growth, magnetization, ac susceptibility, and specific heat, Phys. Rev. B 82, 064404 (2010).
[29] S. V. Grigoriev, V. A. Dyadkin, E. V. Moskvin, D. Lamago, T. Wolf, H. Eckerlebe, and S. V. Maleyev, Helical spin structure of $\mathrm{Mn}_{1-\gamma} \mathrm{Fe}_{y} \mathrm{Si}$ under a magnetic field: Small angle neutron diffraction study, Phys. Rev. B 79, 144417 (2009).

[30] S. V. Grigoriev, E. V. Moskvin, V. A. Dyadkin, D. Lamago, T. Wolf, H. Eckerlebe, and S. V. Maleyev, Chiral criticality in the doped helimagnets $\mathrm{Mn}_{1-y} \mathrm{Fe}_{y} \mathrm{Si}$, Phys. Rev. B 83, 224411 (2011).

[31] C. Franz, F. Freimuth, A. Bauer, R Ritz, C. Schnarr, C. Duvinage, T. Adams, S. Blügel, A. Rosch, Y. Mokrousov, and C. Pfleiderer, Real-Space and Reciprocal-Space Berry Phases in the Hall Effect of $\mathrm{Mn}_{1-x} \mathrm{Fe}_{x} \mathrm{Si}$, Phys. Rev. Lett. 112, 186601 (2014).

[32] L. J. Bannenberg, R. M. Dalgliesh, T. Wolf, F. Weber, and C. Pappas, Evolution of helimagnetic correlations in $\mathrm{Mn}_{1-x} \mathrm{Fe}_{x} \mathrm{Si}$ with doping: A small-angle neutron scattering study, Phys. Rev. B 98, 184431 (2018).

[33] L. J. Bannenberg, F. Weber, A. J. E. Lefering, T. Wolf, and C Pappas, Magnetization and ac susceptibility study of the cubic chiral magnet $\mathrm{Mn}_{1-x} \mathrm{Fe}_{x} \mathrm{Si}$, Phys. Rev. B 98, 184430 (2018).

[34] A. E. Petrova, S. Y. Gavrilkin, D. Menzel, and S. M. Stishov, Physical properties of $\left(\mathrm{Mn}_{1-x} \mathrm{Fe}_{x}\right) \mathrm{Si}$ at $x \simeq 0.15$ along the critical trajectory, Phys. Rev. B 100, 094403 (2019).

[35] S. V. Demishev, A. N. Samarin, V. V. Glushkov, M. I. Gilmanov, I. I. Lobanova, N. A. Samarin, A. V. Semeno, Nikolai E. Sluchanko, N. M. Chubova, V. A. Dyadkin, and S. V. Grigoriev, Anomalous spin relaxation and quantum criticality in $\mathrm{Mn}_{1-x} \mathrm{Fe}_{x} \mathrm{Si}$ solid solutions, JETP Lett. 100, 28 (2014).

[36] S. V. Demishev, A. N. Samarin, J. Huang, V. V. Glushkov, I. I. Lobanova, N. E. Sluchanko, N. M. Chubova, V. A. Dyadkin, S. V. Grigoriev, M. Y. Kagan, J. Vanacken, and V. V. Moshchalkov, Magnetization of $\mathrm{Mn}_{1-x} \mathrm{Fe}_{x} \mathrm{Si}$ in high magnetic fields up to $50 \mathrm{~T}$ : Possible evidence of a field-induced Griffiths phase, JETP Lett. 104, 116 (2016).

[37] J. Kindervater, T. Adams, A. Bauer, F. X. Haslbeck, A. Chacon, S. Mühlbauer, F. Jonietz, A. Neubauer, U. Gasser, G. Nagy, N. Martin, W. Häußler, R. Georgii, M. Garst, and C. Pfleiderer, Evolution of magnetocrystalline anisotropies in $\mathrm{Mn}_{1-x} \mathrm{Fe}_{x} \mathrm{Si}$ and $\mathrm{Mn}_{1-x} \mathrm{Co}_{x} \mathrm{Si}$ as inferred from small-angle neutron scattering and bulk properties, Phys. Rev. B 101, 104406 (2020).

[38] C. Pappas, E. Lelievre-Berna, P. Falus, P. M. Bentley, E. Moskvin, S. Grigoriev, P. Fouquet, and B. Farago, Chiral Paramagnetic Skyrmion-like Phase in MnSi, Phys. Rev. Lett. 102, 197202 (2009).

[39] C. Pappas, E. Lelievre-Berna, P. Bentley, P. Falus, P. Fouquet, and B. Farago, Magnetic fluctuations and correlations in $\mathrm{MnSi}$ : Evidence for a chiral skyrmion spin liquid phase, Phys. Rev. B 83, 224405 (2011).

[40] M. Blume, Polarization effects in the magnetic elastic scattering of slow neutrons, Phys. Rev. 130, 1670 (1963).

[41] S. V. Maleyev, V. G. Baryakhtar, and P. A. Suris, The scattering of slow neutrons by complex magnetic structures, Sov. Phys. Solid State 4, 2533 (1963).

[42] A. P. Murani and F. Mezei, Neutron spin echo study of spin glass dynamics, in Neutron Spin Echo, Lecture Notes in Physics Vol. 128 (Springer, Berlin, 1980), p. 104.

[43] C. Pappas, G. Ehlers, and F. Mezei, Neutron-spin-echo spectroscopy and magnetism, in Neutron Scattering from Magnetic 
Materials, edited by Tapan Chatterji (Elsevier, Amsterdam, 2006), p. 521.

[44] M. Janoschek, M. Garst, A. Bauer, P. Krautscheid, R. Georgii, P. Böni, and C. Pfleiderer, Fluctuation-induced first-order phase transition in Dzyaloshinskii-Moriya helimagnets, Phys. Rev. B 87, 134407 (2013).

[45] S. V. Grigoriev (private communication).

[46] S. V. Grigoriev, D. Chernyshov, V. A. Dyadkin, V. Dmitriev, E. V. Moskvin, D. Lamago, T. Wolf, D. Menzel, J. Schoenes, S. V. Maleyev, and H. Eckerlebe, Interplay between crystalline chirality and magnetic structure in $\mathrm{Mn}_{1-x} \mathrm{Fe}_{x} \mathrm{Si}$, Phys. Rev. B 81, 012408 (2010).

[47] L. J. Bannenberg, K. Kakurai, P. Falus, E. Lelièvre-Berna, R. M. Dalgliesh, C. D. Dewhurst, F. Qian, Y. Onose, Y. Endoh, Y. Tokura, and C. Pappas, Universality of the helimagnetic transition in cubic chiral magnets: Small angle neutron scattering and neutron spin echo spectroscopy studies of FeCoSi, Phys. Rev. B 95, 144433 (2017).

[48] L. J. Bannenberg, A. J. E. Lefering, K. Kakurai, Y. Onose, Y. Endoh, Y. Tokura, and C. Pappas, Magnetic relaxation phenomena in the chiral magnet $\mathrm{Fe}_{1-x} \mathrm{Co}_{x} \mathrm{Si}$ : An ac susceptibility study, Phys. Rev. B 94, 134433 (2016).

[49] M. T. Birch, R. Takagi, S. Seki, M. N. Wilson, F. Kagawa, A. Štefančič, G. Balakrishnan, R. Fan, P. Steadman, C. J. Ottley, M. Crisanti, R. Cubitt, T. Lancaster, Y. Tokura, and P. D. Hatton, Increased lifetime of metastable skyrmions by controlled doping, Phys. Rev. B 100, 014425 (2019).

[50] R. M. Pickup, R. Cywinski, C. Pappas, B. Farago, and P. Fouquet, Generalized Spin-Glass Relaxation, Phys. Rev. Lett. 102, 097202 (2009).

[51] C. Pappas, F. Mezei, G. Ehlers, P. Manuel, and I. A. Campbell, Dynamic scaling in spin glasses, Phys. Rev. B 68, 054431 (2003).

[52] I. E. Dzyaloshinskii, Theory of helicoidal structures in antiferromagnets. I. Nonmetals, Zh. Eksp. Teor. Fiz. 46, 1420 (1964) [Sov. Phys. JETP 19, 960 (1964)].

[53] I. E. Dzyaloshinskii, The theory of helicoidal structures in antiferromagnets. II. Metals, Zh. Eksp. Teor. Fiz. 47, 336 (1965) [Sov. Phys. JETP 20, 223 (1965)].

[54] P. Bak and M. H. Jensen, Theory of helical magnetic structures and phase transitions in MnSi and FeGe, J. Phys. C 13, L881 (1980).

[55] F. Qian, L. J. Bannenberg, H. Wilhelm, G. Chaboussant, L. M. DeBeer-Schmitt, M. P. Schmidt, A. Aqeel, T. T. M. Palstra, E. H. Brück, A. J. E. Lefering, C. Pappas, M. Mostovoy, and A. O. Leonov, New magnetic phase of the chiral skyrmion material $\mathrm{Cu}_{2} \mathrm{OSeO}_{3}$, Sci. Adv. 4, eaat7323 (2018).

[56] T. Tanigaki, K. Shibata, N. Kanazawa, X. Yu, Y. Y. Onose, H. S. Park, D. Shindo, and Y. Tokura, Real-space observation of short-period cubic lattice of skyrmions in MnGe, Nano Lett. 15, 5438 (2015).
[57] Y. Fujishiro, N. Kanazawa, T. Nakajima, X. Z. Yu, K. Ohishi, Y. Kawamura, K. Kakurai, T. Arima, H. Mitamura, A. Miyake, K. Akiba, M. Tokunaga, A. Matsuo, K. Kindo, T. Koretsune, R. Arita, and Y. Tokura, Topological transitions among skyrmionand hedgehog-lattice states in cubic chiral magnets, Nat. Commun. 10, 1059 (2019).

[58] T. T. J. Mutter, A. O. Leonov, and K. Inoue, Skyrmion instabilities and distorted spiral states in a frustrated chiral magnet, Phys. Rev. B 100, 060407(R) (2019).

[59] T. H. R. Skyrme, A non-linear field theory, Proc. R. Soc. London A 260, 127 (1961).

[60] A. O. Leonov and M. Mostovoy, Multiply periodic states and isolated skyrmions in an anisotropic frustrated magnet, Nat. Commun. 6, 8275 (2015)

[61] S. V. Demishev, I. I. Lobanova, V. V. Glushkov, T. V. Ischenko, N. E. Sluchanko, V. A. Dyadkin, N. M. Potapova, and S. V. Grigoriev, Quantum bicriticality in $\mathrm{Mn}_{1-x} \mathrm{Fe}_{x} \mathrm{Si}$ solid solutions: Exchange and percolation effects, JETP Lett. 98, 829 (2013).

[62] V. V. Glushkov, I. I. Lobanova, V. Y. Ivanov, V. V. Voronov, V. A. Dyadkin, N. M. Chubova, S. V. Grigoriev, and S. V. Demishev, Scrutinizing Hall Effect in $\mathrm{Mn}_{1-x} \mathrm{Fe}_{x} \mathrm{Si}$ : Fermi Surface Evolution and Hidden Quantum Criticality, Phys. Rev. Lett. 115, 256601 (2015).

[63] T. Kurumaji, T. Nakajima, M. Hirschberger, A. Kikkawa, Y. Yamasaki, H. Sagayama, H. Nakao, Y. Taguchi, T. Arima, and Y. Tokura, Skyrmion lattice with a giant topological Hall effect in a frustrated triangular-lattice magnet, Science 365, 914 (2019).

[64] S. von Malottki, B. Dupé, P. F. Bessarab, A. Delin, and S. Heinze, Enhanced skyrmion stability due to exchange frustration, Sci. Rep. 9, 12299 (2019).

[65] H. Y. Yuan, O. Gomonay, and Mathias Kläui, Skyrmions and multisublattice helical states in a frustrated chiral magnet, Phys. Rev. B 96, 134415 (2017).

[66] V. Laliena, J. Campo, and Y. Kousaka, Understanding the $H-T$ phase diagram of the monoaxial helimagnet, Phys. Rev. B 94, 094439 (2016).

[67] A. O. Leonov and A. N. Bogdanov, Crossover of skyrmion and helical modulations in noncentrosymmetric ferromagnets, New J. Phys. 20, 043017 (2018).

[68] M. Shinozaki, S. Hoshino, Y. Masaki, A. N. Bogdanov, A. O. Leonov, J.-I. Kishine, and Y. Kato, Fan-type spin structure in uni-axial chiral magnets, arXiv:1705.07778.

[69] Y. A. Kharkov, O. P. Sushkov, and M. Mostovoy, Bound States of Skyrmions and Merons near the Lifshitz Point, Phys. Rev. Lett. 119, 207201 (2017).

[70] B. Schaub and D. Mukamel, Phase diagrams of systems exhibiting incommensurate structures, Phys. Rev. B 32, 6385 (1985).

[71] Data from this work are available at https://doi.ill.fr/10.5291/ ILL-DATA.4-03-1729. 